\title{
A REVIEW OF A RIESZ BASIS PROPERTY FOR INDEFINITE STURM-LIOUVILLE PROBLEMS
}

\author{
PAul Binding AND ANDREas Fleige
}

Abstract. For an indefinite weight function $r$ on $[-1,1]$ with $x r(x)>0$ we consider connections between a Riesz basis property of the indefinite Sturm-Liouville eigenvalue problem

$$
-y^{\prime \prime}=\lambda r y, \quad y(-1)=y(1)=0
$$

and various different conditions, for example HELP-type inequalities

$$
\left(\int_{0}^{1}\left|h^{\prime}\right|^{2} \frac{1}{r} d x\right)^{2} \leqslant k\left(\int_{0}^{1}|h|^{2} d x\right)\left(\int_{0}^{1}\left|\left(\frac{h^{\prime}}{r}\right)^{\prime}\right|^{2} d x\right)
$$

for certain classes of functions $h$ on $[0,1]$. We show that for so-called strongly odd dominated functions $r$ (including odd $r$ ) these problems are equivalent. This allows us to apply known results from the theory of one problem to the others.

Mathematics subject classification (2010): Primary 34B24; Secondary 26D10, 34L10, 47A75. Keywords and phrases: indefinite Sturm-Liouville problem, Riesz basis, HELP inequality.

\section{REFERENCES}

[1] T. AzIzov, I. Iohvidov, Linear operators in Spaces with an Indefinite Metric, Wiley, 1989.

[2] N.L. AbasheEva, S.G. PyatKov, Counterexamples in indefinite Sturm-Liouville problems, Siberian Adv. Math. 7 (1997), 1-8.

[3] R. BEALS, An abstract treatment of some forward-backward problems of transport and scattering, J. Funct. Anal. 34 (1979), 1-20.

[4] R. BEALS, Indefinite Sturm-Liouville problems and half range completeness, J. Differential Equ. 56 (1985), 391-407.

[5] M. Baouendi, P. Grisvard, Sur une équation d'évolution changeant de type, J. Funct. Anal. 2 (1968), 352-367.

[6] C. Bennewitz, The HELP inequality in the regular case, Internat. Schriftenreihe Numer. Math. 80 (1987), 337-346.

[7] P. Binding, B. Ćurgus, A counterexample in Sturm-Liouville completeness theory, Proc. Roy. Soc. Edinburgh A 134 (2004), 244-248.

[8] P. Binding, B. Ćurgus, Riesz Bases of Root Vectors of Indefinite Sturm-Liouville Problems with Eigenparameter Dependent Boundary Conditions, I, Oper. Theory: Adv. Appl. 163 (2006), 75-96.

[9] P. Binding, A. Fleige, Conditions for an indefinite Sturm-Liouville Riesz basis property, Oper. Theory: Adv. Appl. 198 (2009), 87-95.

[10] J. BognáR, Indefinite Inner Product Spaces, Springer, 1974.

[11] K. CASE, Elementary solutions of the transport equation and their applications, Ann. Physics 9 (1960), 1-23.

[12] B. ĆURGUS, On the regularity of the critical point infinity of definitizable operators, Integr. Equ. Oper. Theory 8 (1985), 462-488.

[13] B. ĆURGUS, H. LANGER, A Krein space approach to symmetric ordinary differential operators with an indefinite weight function, J. Differential Equ. 79 (1989), 31-61. 
[14] B. ĆURgus, B. NAJMAn, A Krein space approach to elliptic eigenvalue problems with indefinite weights, Diff. and Integr. Equ. 7 (1994), 1241-1252.

[15] W. D. Evans, W. N. EveritT, A return to the Hardy-Littlewood integral inequality, Proc. Roy. Soc. Lond. A 380 (1982), 447-486.

[16] W. D. Evans, W. N. Everitt, HELP inequalities for limit-circle and regular problems. Proc. Roy. Soc. Lond. A 432 (1991), 367-390.

[17] W. N. EVERITT, On an extension to an integro-differential inequality of Hardy, Littlewood and Polya, Proc. Roy. Soc. Edinburgh A 69 (1972), 295-333.

[18] A. FleIGE, The "turning point condition" of Beals for indefinite Sturm-Liouville problems, Math. Nachr. 172 (1995), 109-112.

[19] A. FLEIGE, Spectral Theory of Indefinite Krein-Feller Differential Operators, Mathematical Research 98, Akademie Verlag, Berlin, 1996.

[20] A. Fleige, A counterexample to completeness properties for indefinite Sturm-Liouville problems, Math. Nachr. 190 (1998), 123-128.

[21] A. Fleige, A Necessary Aspect of the Generalized Beals Condition for the Riesz Basis Property of Indefinite Sturm-Liouville Problems, Oper. Theory: Adv. Appl. 175 (2007), 89-94.

[22] A. Fleige, The Riesz Basis Property of an Indefinite Sturm-Liouville Problem with a Non Odd Weight Function, Integr. Equ. Oper. Theory, Vol. 60 (2008), 237-246.

[23] R. HangelbroeK, Linear analysis and solution of neutron transport problems, Transport Theory Statist. Phys. 5 (1976), 1-85.

[24] G. Hardy, J. LitTlewood, Some integral inequailities connected with the calculus of variations, Quart. J. Math. Oxford 3 (1932), 241-252.

[25] G. Hardy, J. Littlewood, G. Polya, Inequalities, Cambridge, 1934.

[26] H. G. Kaper, M. K. Kwong, C. G. LekKerkerker.,A. Zettl, Full and partial-range eigenfunction expansions for Sturm-Liouville problems with indefinite weights, Proc. Roy. Soc. Edinburgh A 98 (1984), 69-88.

[27] I. Karabash, A. Kostenko, M. Malamud, The similarity problem for J-nonnegative SturmLiouville operators, J. Differential Equ. 246 (2009), 964-997.

[28] H. LANGER, Spectral functions of definitizable operators in Krein spaces, In: D. Butkovic, H. Kraljevic and S. Kurepa (eds.): Functional analysis. Conf. held at Dubrovnik, Yugoslavia, November 2 - 14, 1981. Lecture Notes in Mathematics, Vol. 948, Springer-Verlag, Berlin, Heidelberg, New York, 1982, $1-46$.

[29] C. Pagani, On the parabolic equation $\operatorname{sgn}(x) x^{p} u_{y}-u_{x x}=0$ and a related one. Ann. Mat. Pura Appl. 99 (1974), 333-399.

[30] A.I. PARFenov, On an Embedding Criterion for Interpolation Spaces and Application to Indefinite Spectral Problems, Siberian Mathematical Journal 44 (2003), 638-644.

[31] A.I. Parfenov, On Curgus' condition in indefinite Sturm-Liouville problems, Siberian Adv. Math. 15, (2005), 68-103.

[32] S.G. Pyatkov, Some properties of eigenfunctions of linear sheaves. Siberian Math. J. 30 (1989), 587-597.

[33] S.G. Pyatkov, Certain properties of eigenfunctions of linear pencils, Math. Notes 51 (1992), 90-95.

[34] S.G. PyatKov, Some properties of eigenfunctions and associated functions of indefinite SturmLiouville problems, In the book Nonclassical Equations of Mathematical Physics (in Russian), Sobolev Institute of Mathematics, Novosibirsk, 2005, pp. 240-251.

[35] H. TRIEBEL, Interpolation Theory, Function Spaces, Differential Operators, North-Holland, 1978.

[36] H. VOLKMER, Sturm-Liouville problems with indefinite weights and Everitt's inequality, Proc. Roy. Soc. Edinburgh A 126, 5 (1996), 1097-1112. 